\title{
RELIABILITY STUDIES OF PLANAR SILICON DETECTORS
}

Claus Goessling, Erik H.M. Heije, Pierre Jarron, H. Andrew Parker, Nicola Redaelli, Leonardo Rossi CERN, Geneva, Switzerland

\section{Abstract}

For future large scale, long term applications of silicon particle detectors in high energy physics experiments, e.g. in proton or electron colliders, it is necessary to evaluate the reliability of such detectors. An automated measurement apparatus has been built, which is used to test planar silicon detectors for several days or weeks, both with and without irradiation. Defective detectors were studied with SEM and EBIC microscopy. Special test structures were designed to evaluate and possibly improve detector characteristics.

\section{Introduction}

The radiation environment in which a semiconductor is used is generally detrimental to the semiconductor material. Degradation may also occur in the mounting or in connections. Furthermore, it is not necessarily induced by the irradiation only, but may be related to the high electrical field, or to electrochemical ageing. The study of detector reliability has always been of prime importance in space applications [1], where repair is a remote possibility.

Planar silicon detectors can be produced in large quantities, at relatively low cost [2]. This makes them suitable for application in high energy physics experiments, where often a large detecting area must be equipped. Long term operation, coupled to difficult access conditions, also require in this case a reliable detector functioning. It is anticipated that planar silicon detectors will have a behaviour comparable to that of similar microelectronic circuits, except for effects related to the high resistivity. Details of the manufacturing process generally play an important role in the relative radiation hardness. A test structure is proposed, which can be used to evaluate a given technology, and possibly improve it.

The following sections describe studies of planar detectors from different manufacturers, with various methods, and for several experimental projects at CERN. No definitive conclusions should be drawn from the preliminary data presented here, except that both the detectors and their testing procedures should be further improved. With this aim a fruitful collaboration is maintained with the different manufacturers.

\section{Leakage current instabilities}

The simplest test of a silicon detector is the measurement of its diode reverse current or "leakage current" $\mathrm{I}_{\mathrm{r}}$. The lower this reverse current, the lower can be the shot noise contribution to the detector noise $\left(\alpha \sqrt{ } I_{r}\right)$. In surface barrier detectors the surface leakage current is generally predominant, although it can be stable and well under control by the application of empirical manufacturing methods. In the planar silicon process a thermally grown silicon oxide is used to delineate the ion-implanted diode area and to passivate the surface around it. The preparation of this oxide is equally empirical, although better documented [3]. However, significantly lower reverse currents can be achieved with the planar process, approaching the limiting current set by the generationrecombination of carriers in the bulk of the diode. Kemmer [4] reported a current of $2 \mathrm{nA} \mathrm{cm}-2$ or $70 \mathrm{nA} \mathrm{cm}-3$ at total depletion. The best photodiodes show similar or even lower reverse currents, e.g. in the photodiode 51790 the reverse current is typically $0.5-1 \mathrm{nA} \mathrm{cm}-2$ or $25-50 \mathrm{nA} \mathrm{cm}^{-3}$ (supposing a $200 \mu \mathrm{m}$ thick depletion region in this device) [5].

\subsection{Spatial non-uniformity of current}

These low leakage currents are usually reported for "typical" selected diodes of limited size, e.g. $1 \mathrm{~cm}^{2}$ of surface area. For applications in high energy physics it is essential to obtain a stable, low current over a large area of silicon. The average current for a large detector $\left(30 \mathrm{~cm}^{2}\right)$ is more than proportional, due to "bad spots". In silicon microstrip detectors such "bad spots" cause "leaky" strips. The identification of "bad spots" either with native defects in the silicon or with process induced defects is studied in sect. 5 .

For use in a vertex detector system in the Omega $\left(\Omega^{\prime}\right)$ spectrometer $10 /$ microstrip detectors, each with 512 strips of $50 \mu \mathrm{m}$ width, were purchased. The testing of these detectors was done with the test bench designed by Jarron [6] and described in sect. 3. Several observations could be made. Some pairs of strips are not well separated, and although signals can be obtained, there is a loss of position information. Other strips are bad because they are not connected, or they have a current $>.01 \mu \mathrm{A}$ $\left(=.8 \mu \mathrm{A} \mathrm{cm}^{-1}\right)$. In fig. 1 the number of bad strips on these 10 detectors is shown. Leaky strips often occur in clusters. It is observed that such clusters may grow with time: we call it the "oil-drop" effect, because the current spreads to neighbouring strips if the bad strip is disconnected, such as oil which one tries to clean with water. A possible explanation will be given in sect. 5. A detector is unusable if the current is so large that the signals are hidden by the current shot noise or if total depletion of the volume cannot be achieved (voltage drop over $100 \mathrm{k} \Omega$ bias resistor).

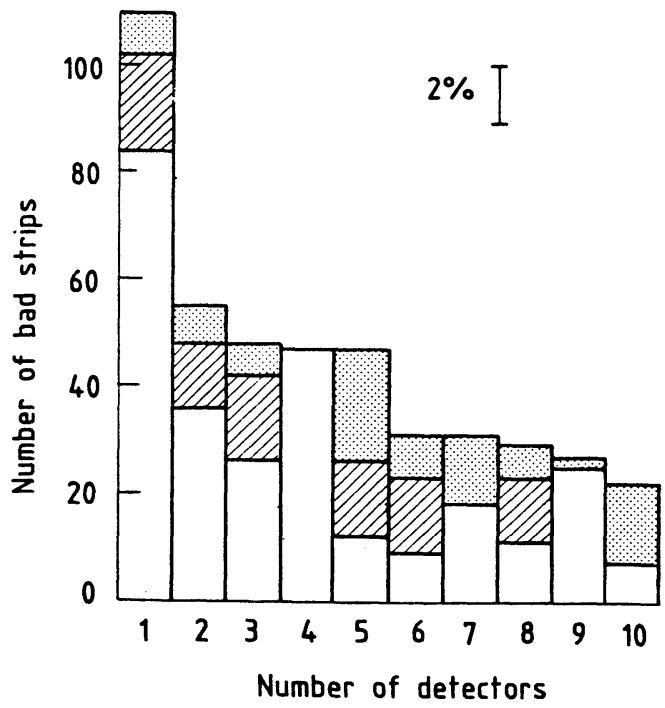

Pig. 1 The number of bad strips in 10 microstrip detectors. For each 512 strip detector are indicated: the not connected strips (problems in mounting or bonding) below; the number of strips with short circuits between them (hatched, in the middle); and the strips with leakage current $>.1$ uA (the top of each colums). 


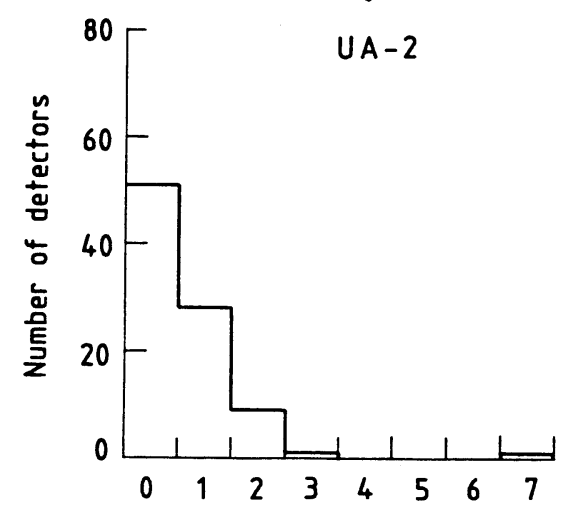

Number of bad segments

Fig. 2 Distribution of the number of bad segments for 90 detectors. $A$ bad segment has a leakage current >0.4 $\mu \mathrm{A}$.

For use in the UA2 proton-antiproton collider experiment 432 segmented detectors have to be mounted, each with a sensitive area of $24.4 \mathrm{~cm}^{2}$, and totalling $1.05 \mathrm{~m}^{2}$. The detector consists of 7 segments of $349 \mathrm{~mm}^{2}$ each, separated by an oxide wall $100 \mu \mathrm{m}$ wide and $.6 \mu \mathrm{m}$ thick. These detectors are tested on another test bench, also to be described in sect. 3 . For 90 tested detectors, the "bad-segment" distribution is shown in fig. 2. A bad segment is defined as one which has a current $>.4 \mu \mathrm{A}$ $\left(=.11 \mu \mathrm{A} \mathrm{cm}^{-2}\right)$. Some of these detectors have been made with a common guard ring around the segments. This did not reduce the occurrence of bad segments, and a bad segment could well be found inside a guard ring, itself with very low current. This shows that high leakage is not necessarily edge current in the case of a planar detector.

\subsection{Time-dependent current instability}

One may observe an initially low detector current which gradually increases over a period of several hours. It was found that this characteristic often is related to air humidity, and then it can be avoided by operating the detector in a dry nitrogen atmosphere.

Some planar detectors exhibit large initial current and low breakdown voltage, which improve gradually during $1-2 \mathrm{~h}$. After turning off the bias voltage, current of opposite polarity (a few $\mu A$ ) flows during several minutes, showing that a charge of $10^{14}-10^{16}$ carriers has been stored somewhere in the device. In fig. 3 an example is shown of

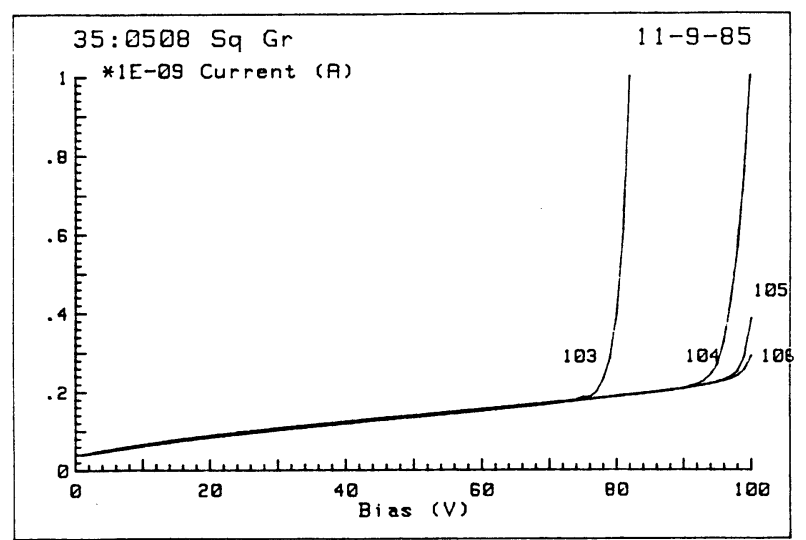

Fig. 3 Reverse current characteristic of a $\sim 1 \mathrm{~mm}^{2}$ square diode on the test chip. The curves were measured consecutively as indicated $(103,104,105$. 106) and each measurement took $\sim 2 \mathrm{mtn}$. The breakdown voltage 1mproves from $78 \mathrm{~V}$ to $\sim 100 \mathrm{~V}$. At total depletion $(\sim 40 \mathrm{~V})$ the current is $\sim 10 \mathrm{nA} \mathrm{cm}-2$ or $\sim 200 \mathrm{nA} \mathrm{cm}^{-3}$ gradually improving breakdown voltage (the time between measurements is $\sim 2$ min.). Sometimes this effect is called "self-healing", and it might be explained by trapping of hot electrons in the oxide around the edge of the device. This trapped charge improves the field profile at the junction curvature. A systematic study of this effect can be made with an appropriate test structure, prepared under well-known process conditions. The detector itself should be equipped with a MOS capacitor and a field-effect device, such as described in sect. 4 .

In earlier studies of silicon detector reliability [7] it was found that the insulating epoxy may become conductive after $1-2$ years. Curing at $120^{\circ} \mathrm{C}$ restored the insulating property. This shows that one should always consider mounting and connections as a possible source of leakage current.

\subsection{Radiation induced current increase in a beam}

Radiation damage occurs in the semiconductor bulk via the introduction of defects, which act as generationrecombination centres, and as charge trapping centres. Therefore, the leakage current increases and the charge collection time or signal rise time may be degraded. Also, an apparent change of the bulk resistivity has been observed in the course of irradiation [7-9].

In this paper, we will focus on a different phenomenon, observed after a relatively short intense irradiation of $\geq 10^{5}$ particles $\mathrm{cm}^{-2} \mathrm{~s}^{-1}$. In fig. 4 are shown the current evolutions of typical "good", and "bad" microstrip detectors, of 520 strips and dimensions $26 \times 26 \mathrm{~mm}^{2}$. As long as the beam intensity is $10^{5}$ minimum ionizing particles on $24 \mathrm{~mm}^{2}$ per burst ( $2.4 \mathrm{~s}$ every $14.4 \mathrm{~s}$ ) the currents remain stable in both detectors. As soon as the beam intensity is tripled, both detectors show an increasing leakage current. After the irradiation, the currents return to their original values in a couple of days. In microstrip detectors from a different manufacturer this current increase was not observed at even higher beam intensity.

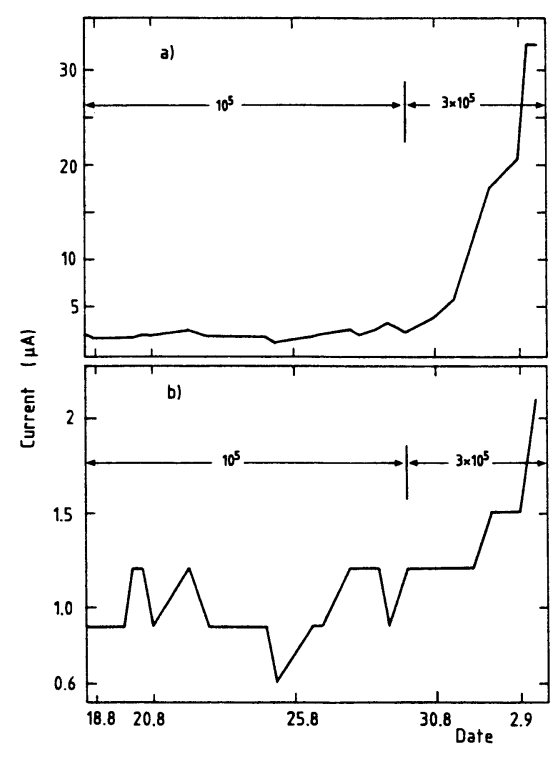

F1g. 4 The current evolution of a "good" microstrip detector (lower curve), and a "bad" one. The current remains stable over days with a beam intensity of $10^{5}$ particles per burst. Shortly after the intensity is increased to $3 \times 10^{5} \mathrm{ppb}$ both currents begin to increase. 


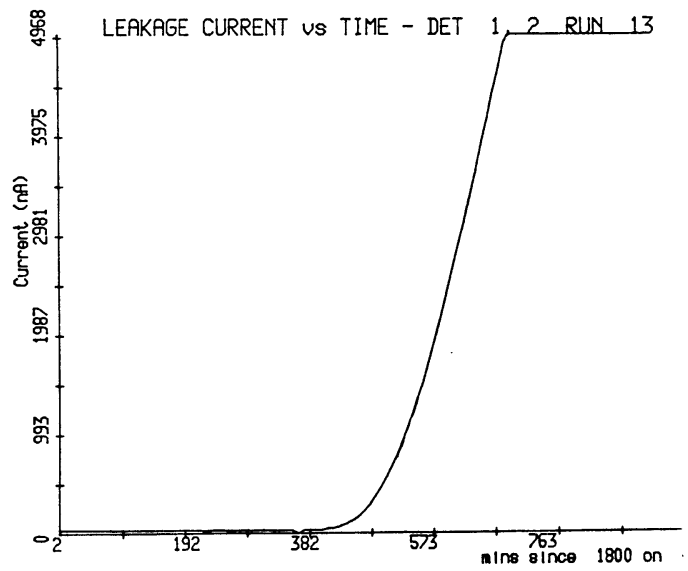

Fig. 5 Reverse current as a function of time during uncollimated irradiation of a $10 \times 10 \mathrm{~mm}^{2}$ planar detector. The blas voltage during irradiation was $80 \mathrm{~V}$. After $\sim 7 \mathrm{~h}$ the current increases from $10 \mathrm{nA}$ to several $\mu A$.

\subsection{Radiation induced current increase, using a source}

The effect can be reproduced by irradiating the microstrip detector with a strong radioactive source $(1 \mathrm{mCi}$ of $905 \mathrm{r}$ ). The source is collimated so that the intensity is $6 \times 10^{6} \mathrm{~cm}^{-2} \mathrm{~s}^{-1}$ in a central spot of $\$ 4 \mathrm{~mm}$, but scattered electrons can reach other parts of the $26 \times 26 \mathrm{~mm}^{2}$ detector. This intensity is equivalent to a beam intensity of $10^{7}$ particles per burst. With this higher intensity, the increase of reverse current occurs already after a few hours if the source is positioned above the centre of the detector. If the source is above the side of the detector, where the aluminium wire bondings are located, the degradation is still faster and takes only $30 \mathrm{~min}$.

The test bench enables monitoring of the current in all strips separately, and it is noted that some strips draw more current than others. After irradiation the current more current than others. After irradiation the current
decreases again and at room temperature recovery is complete after a few days. Heating the detector to $60^{\circ} \mathrm{C}$ shortens the annealing time to a few hours.

\subsection{The "inverted" microstrip detector}

In an attempt to discover the source of the increasing current under intense irradiation different detector structures were tested in the same set-up. The first was a normal $20 \times 20 \mathrm{~mm}^{2}$ ion implanted planar diode detector, which did not show any current change under irradiation. A second detector of $10 \times 10 \mathrm{~mm}^{2}$ also did not show an increase, if irradiated with a collimated source. Using the same source uncollimated the radiation could touch on the oxide edge, and in this case the reverse current started to increase after $\sim 7 \mathrm{~h}$ (fig. 5). Finally, an "inverted" microstrip detector was tested with a plain rectifying contact $\left(26 \times 26 \mathrm{~mm}^{2}\right)$ and 520 ohmic rear contacts in the form of the strip pattern. In this inverted detector no current increase has been observed during a 10-day source irradiation. A part of the measurement is reproduced in fig. 6 .

From these observations it can be concluded that the current increase is related to the irradiation of the oxide. Monitoring of the oxide charge with a MOS capacitor will be essential to understand the mechanism.

The detection efficiency of an inverse structure operated at full depletion is similar to that of a normal detector. This was noted already in 1980 for surface barrier microstrip detectors [10] and verified once more for this planar inverted detector. Such a structure can therefore be used in a high intensity beam.

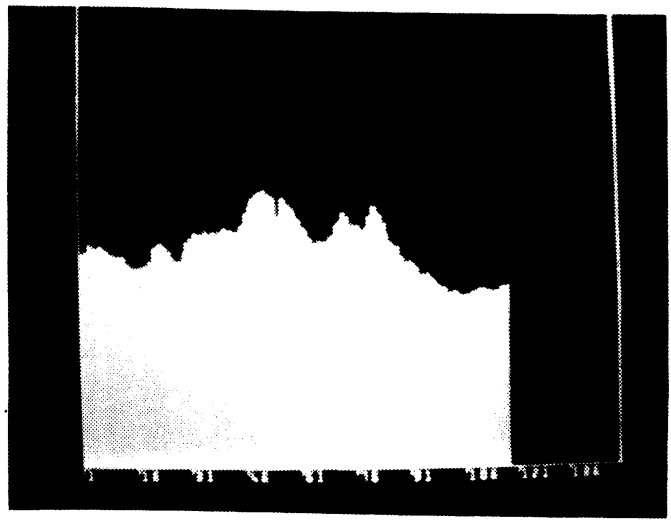

F19. 6 Current evolution with time of the inverted microstrip structure during irradiation. Measurements are taken every hour, this graph represents the last 5 days of a 10-day period. The maximum current is $\sim 16 \mu \mathrm{A}$

\section{Detector test facilities}

For the 520 microstrip detector and for the 7-segment detector separate test facilities were built. Both operate on the same principle, with a LF 355 operational amplifier as current to voltage converter, as shown in fig. 7 . The rectifying contacts are grounded via the input impedance $Z$ of these amplifiers and the reverse bias voltage $V_{B}$ is applied for all detectors at the rear. A current-voltage characteristic is obtained by varying $V_{B}$ and measuring with a CAMAC ADC the amplifier output voltage which is proportional to the reverse current $I_{r}$ in the strip or segment. The full current range is $5 \mu \mathrm{A}$ for a single element (UA2) or $1 \mu \mathrm{A}$ for a single microstrip, with $0.5 \mathrm{nA}$ precision. $A$ display of the $I_{r}-V_{B}$ curves is shown in fig. 8 . Figs 5 and 6 showed different displays of current, also obtained with this system.

For the capacitance measurement, a Radio Frequency (RF) sinusoidal signal $V_{T}$ of $0.5 \mathrm{~V}$, with a frequency $f=10 \mathrm{kHz}$ is also applied at the rear side of the detector via a bias capacitor $C_{B}$. This modulation of the reverse bias voltage creates an additional sinusoidal current $\mathrm{I}_{\mathrm{C}}$ in the detector diode.

$$
I_{C}=V_{T} /\left(Z+1 / j 2 \pi f C_{S}\right) \simeq V_{T} j 2 \pi f C_{S} .
$$

For a diode capacitance $\mathrm{C}_{\mathrm{s}}=120 \mathrm{pF}$ the impedance $Z$ of the amplifier can be neglected.

TEST SYSTEM FOR SLLLCON 7-SEGMENT DETECTORS

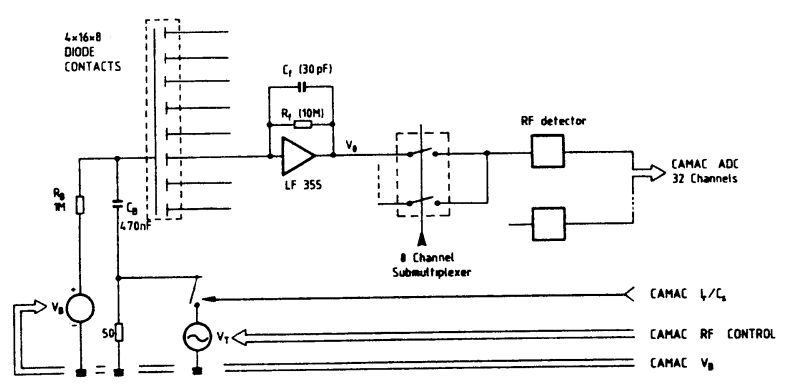

Fig. 7 Schematic of the testing system for the 7-segment (+ guard ring) detectors. The reverse detector blas $V_{B}$ is applied at the rear side of 16 detectors at a time. Bach segment is connected to an operational amplifier LF 355. Bither the current or the capacitance can be measured as a function of $v_{B}$. $v_{B}$, the $R F$ signal $v_{T}(10 \mathrm{kHz})$ and the switch are CAMAC controlled. 


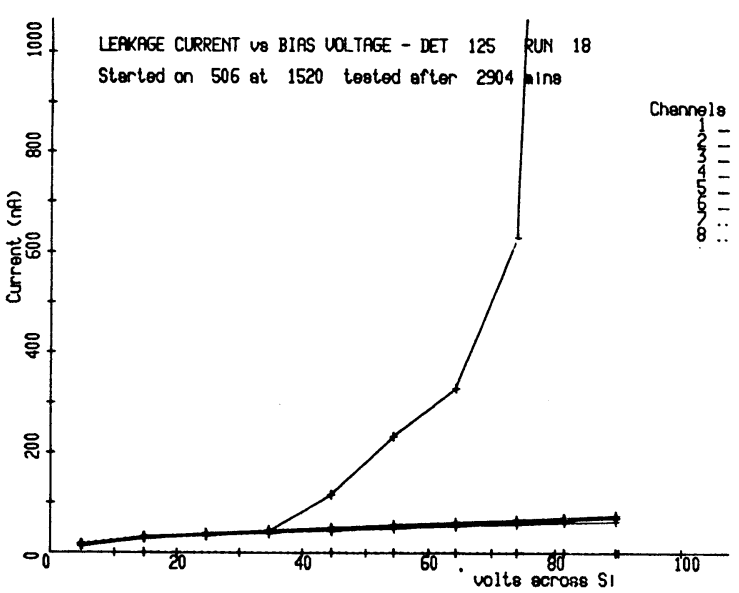

Fig. 8 Graph of the $I_{I}-V_{B}$ characteristic of the 7 segments + guard ring of a 7 segment planar detector. Most segments have a stable current of $\sim 70 \mathrm{nA}$, but one (\#5) shows breakdown above $40 \mathrm{~V}$. In the original, the curves have different colours.
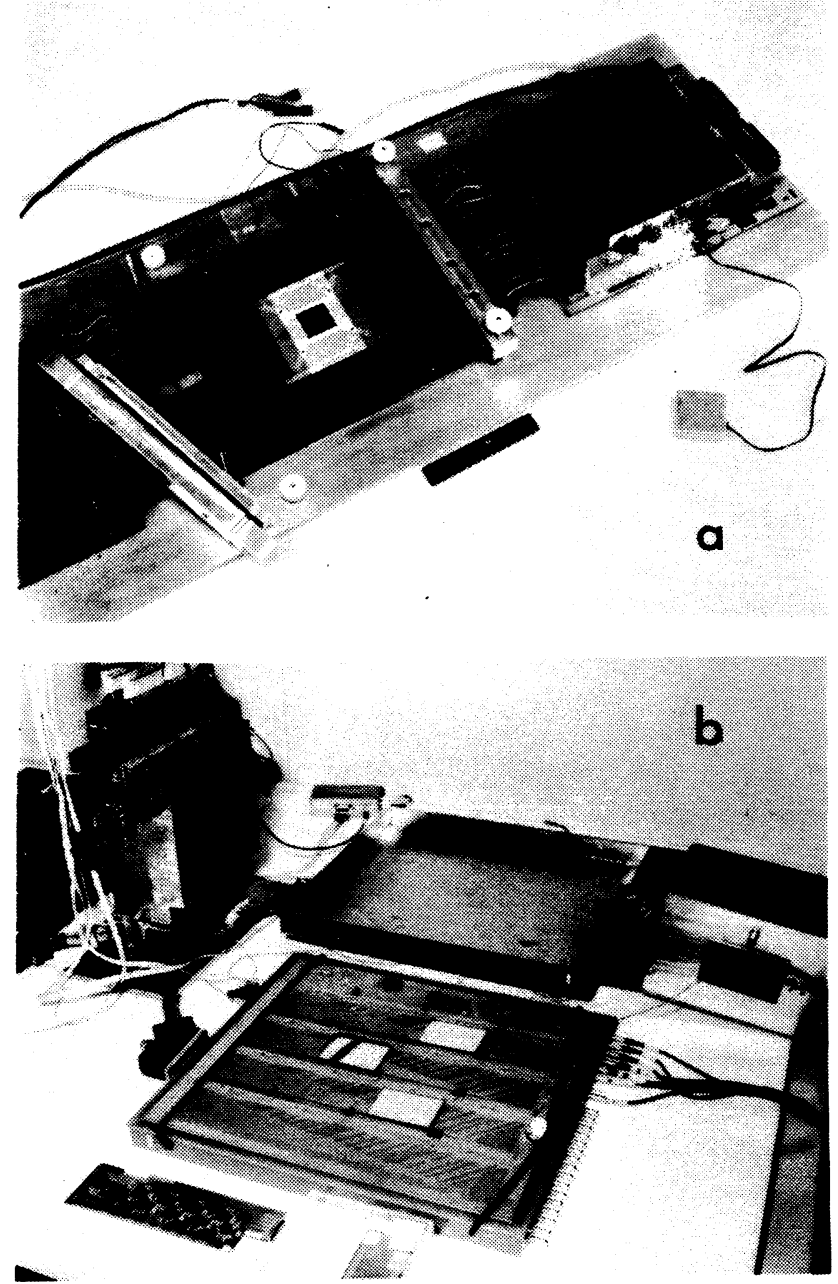

Fig. 10 (a) Test bench for microstrip detector with 512 elements. (b) Test bench for segmented detectors: the picture shows 3 detectors installed on one of the four boards.

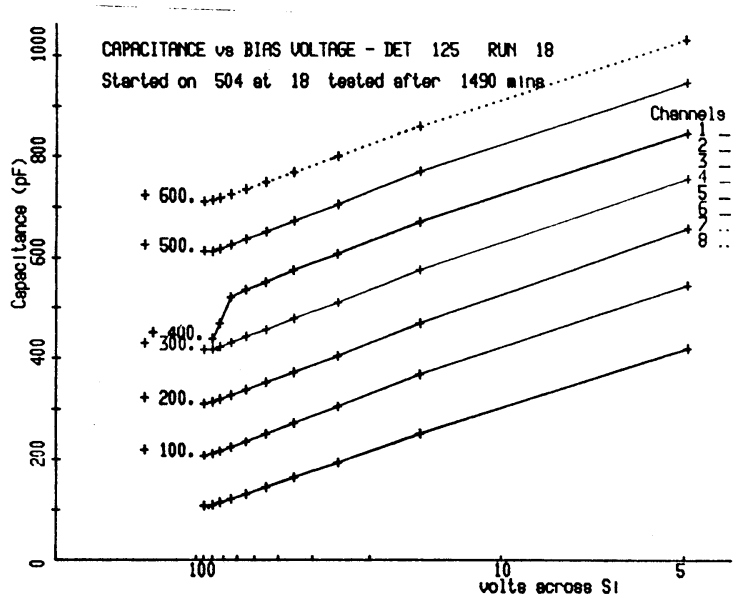

F1q. 9 Graph of the $C_{S}-V_{B}$ characteristic of the 7 segments. The total depletion voltage can be easily determined in this way. To avold overlap of all curves, $100 \mathrm{pF}, 200 \mathrm{pF}$, etc. is added to the curves 2, 3, etc. The data correspond to those of $\mathrm{fig} .8$ The segment with the breakdown (\#5) shows an incorrect capacitance value beyond $80 \mathrm{~V}$, because of the high current.

The additional current can be calibrated and once the DC current $I_{r}$ is measured, the diode capacitance $C_{S}$ can be calculated from the measurement of $\mathrm{I}_{r}+\mathrm{I}_{\mathrm{C}}$. The CAMAC controlled set-up allows this to be done for successive values of $V_{B}$, so that a $C_{S}-V_{B}$ curve can be obtained as shown in fig. 9. From this curve both the silicon resistivity and the total depletion voltage can be determined.

In figs $10(a)$ and $10(b)$ the test bench facilities are shown. On the first bench one microstrip detector with 512 strips can be tested. In the second facility one can measure at one time 4 boards with 16 detectors each, altogether also 512 segments.

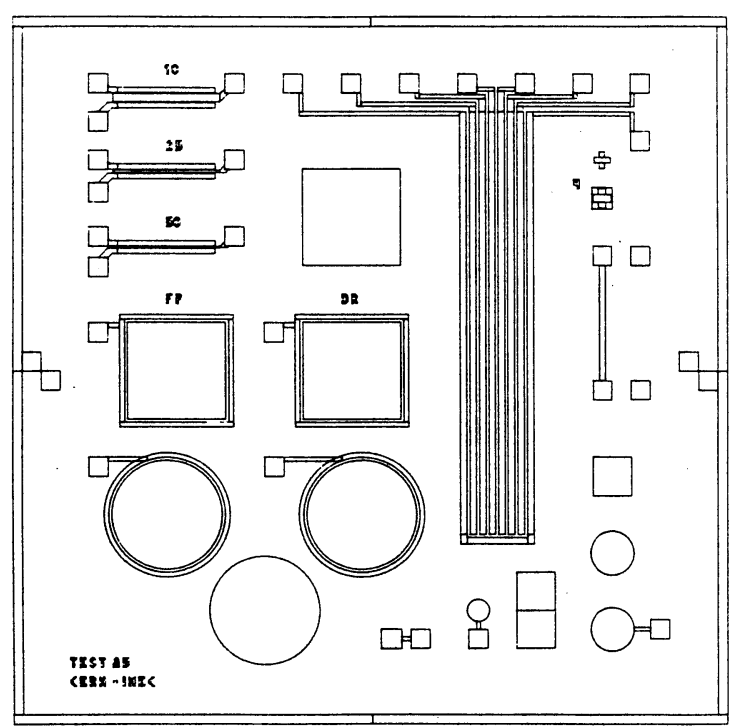

F19. 11 Metallization mask level of the detector test chip. The bonding pads are $100 \times 100 \mathrm{\mu m}^{2}$, the total size is $3.7 \times 3.7 \mathrm{~mm}^{2}$. 


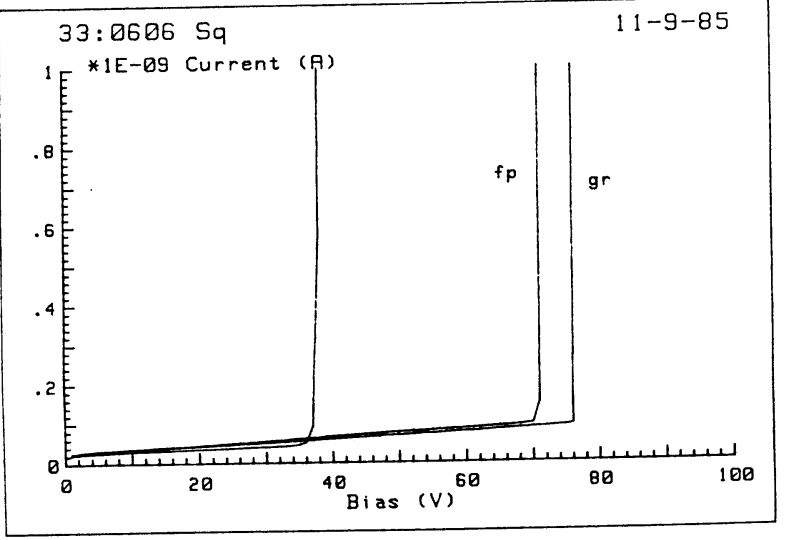

Fig. 12 Comparison of reverse current vs. voltage for 3 different diode structures. The diode with guard ring (gr) has the highest breakdown voltage.

\section{Design of a test chip}

An experimental design of a test chip with various structures was made, as shown in fig. 11 . It contains 11 ion-implanted diodes, both square and circular, of 3 sizes in the relation $1: 4: 25$. The $.25 \mathrm{~mm}^{2}$ diodes are either normal $(n)$ or they are surrounded by an implanted guard ring (gr or $D R$ ) or by a field plate (fp). The breakdown voltage of the diodes is most improved by the guard ring; as shown in fig. 12. The small diodes are in fact not very useful as the lateral extension of the depletion region is too large $(\sim 300 \mu \mathrm{m})$. In this respect even the large diodes are still too small to do accurate measurements, given the very small currents. The detection characteristics are quite acceptable. The FWHM of a $5>\mathrm{Co}$ spectrum (122 keV) is $3.5 \mathrm{keV}$.

An important element is the MOS capacitor of $.08 \mathrm{~mm}^{2}$. It can be used to determine various properties of the oxide, the interface and the bulk [3]. The flat band voltage can be determined from a MOS $\mathrm{C}-\mathrm{V}$ curve as shown in fig. 13. Its value is indicative for the quality of the processing, as unintentional oxide or interface charge will cause a shift to more negative values. The difference in curves (a) and (b) in fig. 13 is caused by the difference in interface charge for substrate orientations $\langle 100\rangle$ and $\langle 111\rangle$

Care is needed to interpret $C-V$ results on this high resistivity silicon. We found that measurements at $100 \mathrm{kHz}$ and $10 \mathrm{kHz}$ give similar results, but at the usual frequency of $1 \mathrm{MHz}$ strong reductions in the capacitance values are found, related to the low carrier concentrations. The low frequency quasistatic $C-V$ curve confirms the values found at $100 \mathrm{kHz}$. Minority carrier lifetime could not be measured with the MOS structure, because inversion is instantaneously obtained, without going into deep depletion. The source of minority carriers is most likely the rear contact, which is close to the depletion region (in terms of the Debye length of 30-40 $\mu \mathrm{m}$ ).

A microstrip structure of 6 strips + guard strip is made, which should enable to study further the irradiation effect discussed in sect. 2.2. However, it might be that this current instability is related to defects, stochastically occurring along the oxide edges. The total length of oxide edge in the test structure is only $32 \mathrm{~mm}$ whereas in the 512 strip detector it is $\sim 27 \mathrm{~m}$. So the test structure would be insensitive to the effect.

Finally there are 3 transistor-like structures which can be used to study the interstrip oxide and interface region but they also function as transistor, as shown in fig. 14. Even with a width of $50 \mu \mathrm{m}$ one observes punch-through, as in a VLSI short-channel device.

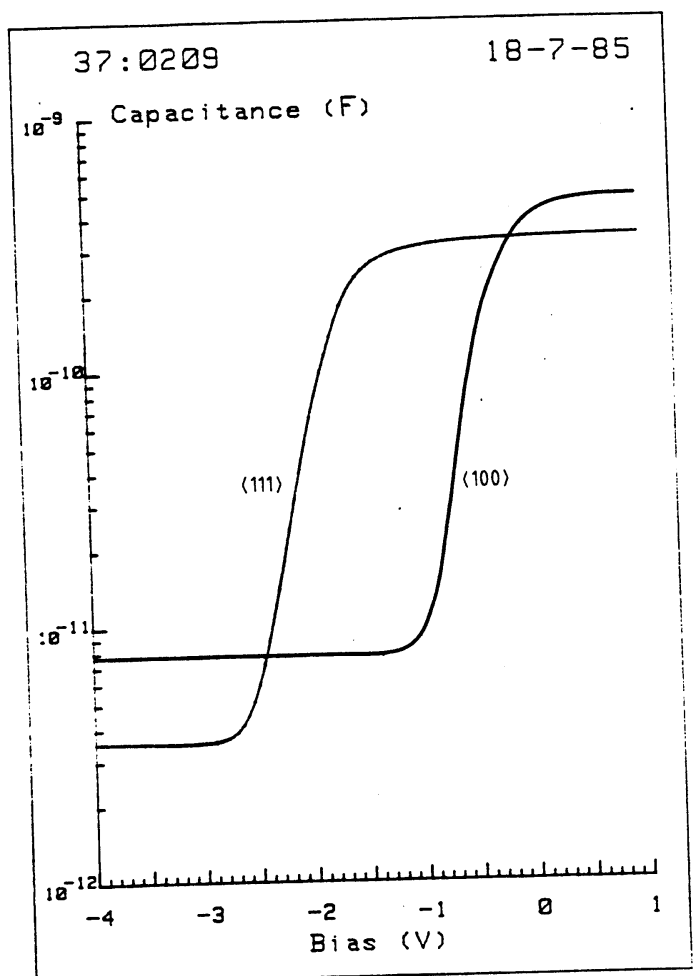

19. $13 \mathrm{C}-\mathrm{V}$ curves $(100 \mathrm{kHz})$ for a $.08 \mathrm{~mm}^{2} \mathrm{MOS}$

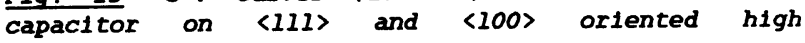
resistivity substrates.

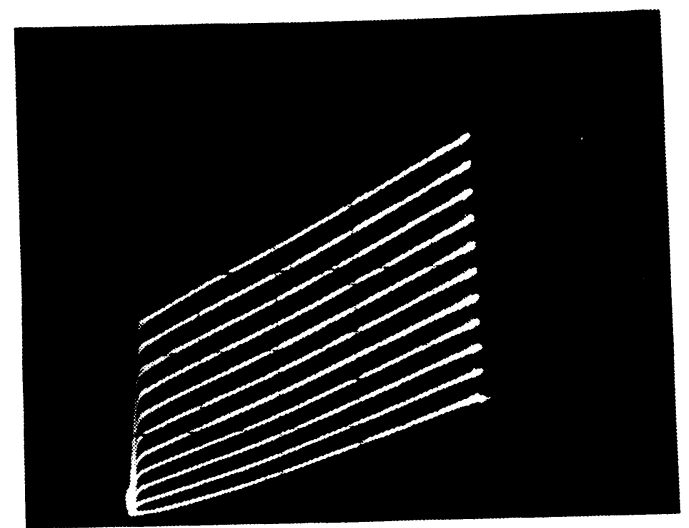

Fiq. $14 I_{D S}-V_{D S}$ transistor characteristics of a test transistor on the high resistivity test chip. Horizontal division $2 \mathrm{~V}$, vertical division $.5 \mathrm{~mA}$. The threshold voltage is $3.3 \mathrm{~V}$ and the step on the gate voltage is $2 \mathrm{~V}$. The transconductance $g_{m}$ is 70 นv.

The purpose of the test chip is to enable accelerated ageing or irradiation of several samples to evaluate the reliability, without damaging the equivalent detectors themselves. Also, small test structures of standard size could be added onto the detectors themselves, to detect in which part of the device degradation occurs, e.g. in the oxide or in the bulk. 


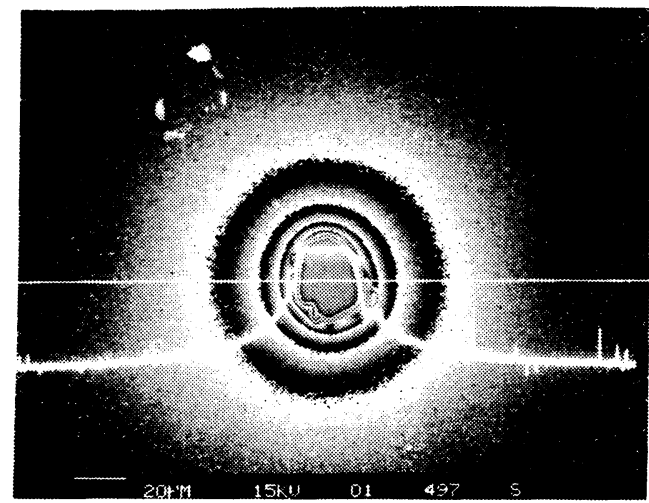

(a)

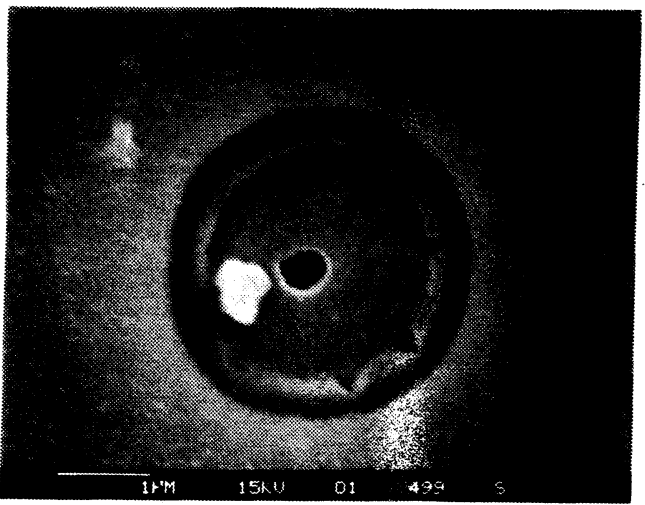

(b)

E19. 15 (a) EBIC picture of part of a leaky segment. The white line is the measured current profile along one cross section of the total picture. The alternance of black/white contours is artificially created to enhance the contrast. (b) on the reverse side of the segment a tiny defect could be found, only $0.5 \mathrm{\mu m}$ large.

\section{EBIC analysis with a Scanning Electron Microscope}

In an attempt to find the "bad spot" sources of high reverse current in some detector elements the Electron Beam Induced Current (EBIC) mode of a Scanning Electron Microscope (SEM) has been employed both on microstrip detectors and on 7-segment detectors. For this EBIC analysis a special large field SEM with large sample chamber was used. The electron beam (.8-40 keV) scans the rear side of the detector while the current in one or several elements is fed into the EBIC amplifier. One may apply some reverse bias voltage to these elements. The signal current is in fact created by holes which traverse the diode structure, thus probing its internal structure. If the electron beam touches a region with high recombination, no holes will be collected and it appears as a white spot. In fig. 15(a) such a white spot is seen (the artificial contrast enhancement creates the alternance of black/white rings), which was found easily in a bad segment of a detector. It was less easy to find the (probably) corresponding defect on the front side of the diode (fig. 15(b)), using the microscope in normal SEM mode. The size of the defect is only a fraction of a $\mu \mathrm{m}$. However, if the total observed segment current $(3 \mu \mathrm{A})$ passes through this defect, a current density of $\sim 10^{2} \mathrm{~A} \mathrm{~cm}^{-2}$ may explain the appearance of this defect, which looks like a molten crater. Such a defect might be observable in Infra-Red (IR) microscopy, but its small size necessitates a high resolution IR microscope. This defect might be related to processing conditions, but could also be initiated by a defect existing on the starting Si-material.
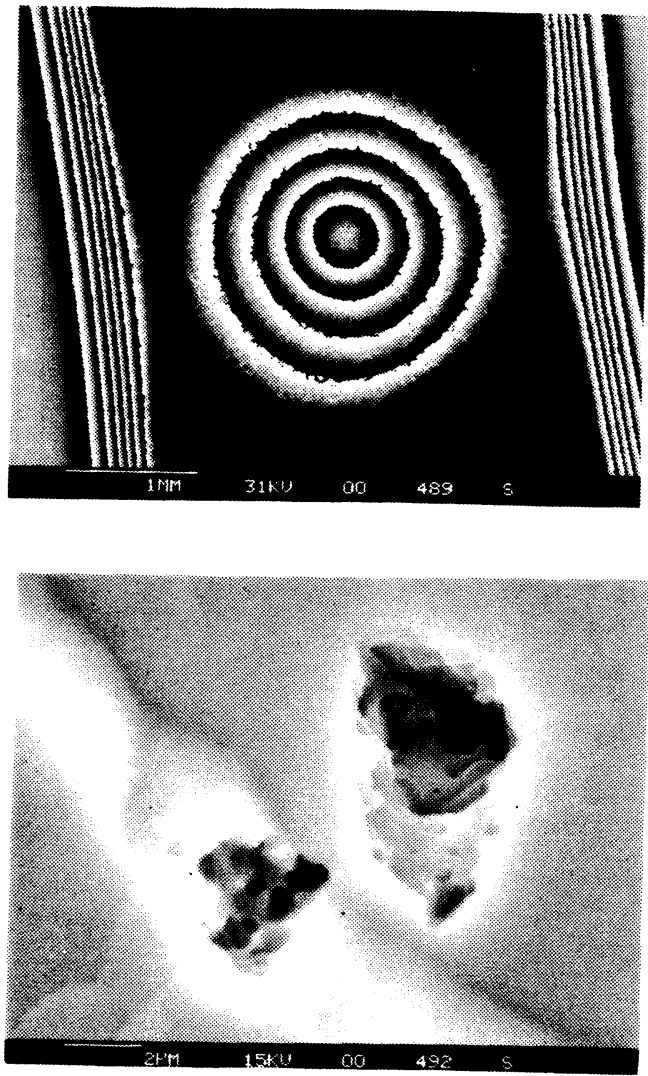

F19. 16 (a) BBIC picture of a region of 80 microstrips of $50 \mathrm{\mu m}$ pltch. The bad spot seems to be spread out over many elements. (b) In reality (SBM) only a tiny defect cluster $(\sim 10 \mu \mathrm{m})$ is found in the centre. It looks l1ke a deep hole and affects the current over many strips ("o11 drop" effect).

A second example of EBIC analysis is shown in fig. 16. On a part of a microstrip detector, the EBIC signal is collected on $\mathbf{8 0}$ strips together. The bad spot occupies nearly all the area, but the defect which is found at the centre, using SEM, is also very small in this case. The hole, with a lot of facets, is found at the rear side of the detector, at the same side where the EBIC is generated. This side of the detector is the lapped side of the original wafer, and was not polished, nor etched. If this defect is responsible for the large current in the strip, it could explain the "oil-drop" effect: by disconnecting one diode at the front, the current caused by the defect on the rear would simply be displaced to adjacent strips.

In fig. 17 a picture is shown of the EBIC scan for a detector segment which has a reverse current ( $300 \mathrm{nA}$ ) which is only slightly higher than the normal $70 \mathrm{nA}$. Again, white regions indicate high recombination/current. Several observations can be made: there exist extended regions with higher current, as in the lower left corner. Around the edges the current is also enhanced. This is partially an artefact, because in this case the adjacent segments are not connected. But if these are connected, one still observes reduced collection at the edges. Finally, a number of big and small white spots are visible, and each is related to some defect structure. For this segment, none of these cause an excessive current, like the ones in figs. 15 and 16. 


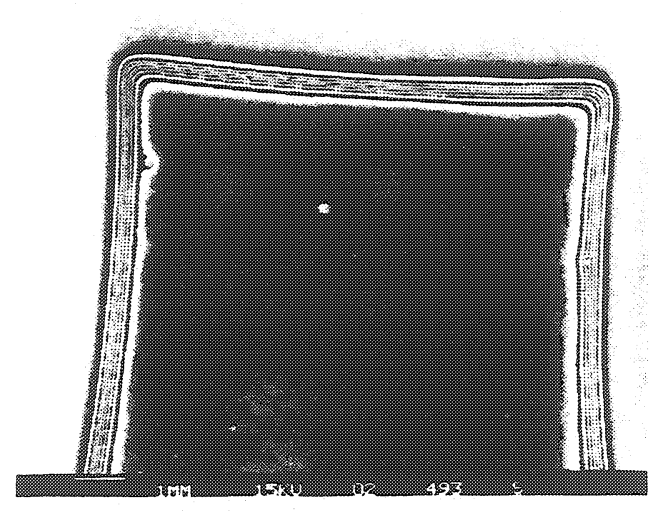

F19. 17 ISIC picture of a large Eraction of one segrent on a 7-segwent detector. Fhe whlte spots are regions of bad collection of the raIC \$lgnal. corresponding to reglons of large carrlerreconbination. and large leakage current.

Further study of these clearly visible defects might be possible, using elemental analysis with induced $X$-rays, ion-backscattering techniques, or other high resolution analysis methods.

From these first EBIC measurements it may already be concluded that large leakage currents are often caused by defects in the diode. rather than in the edges, although recombination in the edges and between segments is always higher than in the diode area. Both defects shown were probably existing already in the original wafer. although they may have been enlarged in processing.

\section{Conclusion}

Work has started in various directions to study problems which are encountered when planar detectors are used for large scale experiments. These problems generally do not preclude the use of these detectors for the aimed purpose, but longer lifetime, better performance and cost reduction could be achieved if the problems were solved.

Thanks to methods developed already in the field of microelectronics many of these problems may be understood, once appropriate measurements have been made.

\section{Acknowledoements}

In the course of this work we arofiting from the appreciated support of professionals from the Interuniversity Microelsctronics Center IMEC in Leuven, Belgium and from the Centre Suisse d'Electronique te Microtechnique CSEM in Neuchatel. Switzarland. Especially. we would like to thank G. Doclerck.I. Debusschers, R. Guye and 3. Jeanneret for many discussions.

The fruitul collaboration with the industrial Canberra Semiconductor N.V.s EnertecSchumbsrger S.A. and Mieron Semiconductor Lto is acknowledged, and it is axpected that the continuation of this work can help to astablish excellent records for planar sllicon datectors.

\section{References}

(11) 8.g. V.A.J. van Lint, G. Gigas and 3. Barengoltz. Correlation of displacement effects produced by electrons, protons and neutrons in silicon. IEEE Trans. Nucl. Sc. NS-22 (1975) 2663.

[2] J.Kemmer, P. Burger, R. Henck and E. Heijne, Parformance and applications of passivated ion-implanted silicon detectors. IEEE Trans. Nucl. Sc. NS-29 (1982) 733.

[3] The most comprehensive review of the oxide-silicon system can be found in: E.H. Nicollian and J.R. Brews, mos (Matal Oxide Semiconductor) Physics and Technology". J. Wiley \& Sons, New York, 1982.

[4] J. Kemmer. Improvement of detector fabrication by the planar process, Nucl. Instr. Math. 226 (1984) 89.

[5] Z. Bian, J. Dobbins and N. Mistry. The use of silicon photodiodes in CSI(TI) calorimster. NuCl. Instr. Meth. A239 (1985) 518

[6] Erik H.M. Hoijne et al. Development of hardware around a silicon microstrip detector with 512 elements, Nucl. Instr. Meth. $226(1984) 63$.

[7] Erik H.M. Heine, Radiation damage: experience with sllicon detectors in high energy particle beams at CERN, procesdings Miniaturization of Detectors, Pisa Conference 1980. Ed. A. Stefanini. Plenum Press, London 1983. Also in: Muon flux measursment with silicon detectors in the CERN noutrino beams, CERN yollow report $83-06$, chapter 5.

[8] 8. Sigfridsson and J.L. Lindström, Electrical properties of electron-irradiated n-type silicon, J. Appl. Phys. 47 (1976) 4611.

[9] H. Dietl, T. Gooch, R. Klanner, M. Pepé and F. Wickens, Radiation damage in silicon strip detectors, preprint. contribution to Europhysics Conf. on High Energy Physics, Bari. 1985.

[10] E.H.M. Haijne et al.o A silicon surface barrier microstrip detector designed for high energy physics experiments. Nucl. Instr. Meth. 178 (1980) 331. 


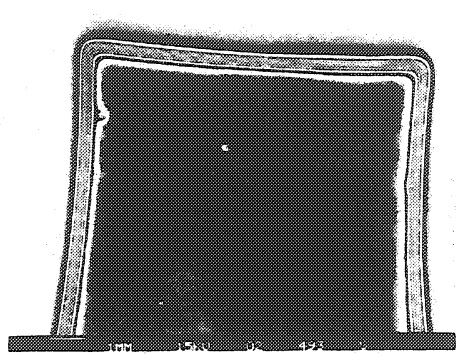

F19. 17 ESIC picture of a large fraction of one segment on a 7-segaent detector. The white spots are reglons of bed collection af the ERIC signal,
corresponding to regions af large carrierrecomblnation, and large leakage current.

Further study of these clearly visible defects might be possible, using elemental analysis with induced $X$-rays,
ion-backscattering techniques, or other high resolution analysis methods.

From these first EBIC measurements it may already be concluded that large leakage currents are often caused by defects in the droda, rather than in the edges, although recor that in the tho adgen alway higher thin in thry may have been enlarged in processing.

\section{Conclusion}

Work has started in various directions to study problems which are encountered when planar detectors are used for large scale experiments. These problems generally do not preciude the use of these detactors for the aimed

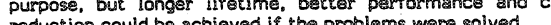

Thanks to methods developed already in the field understood, once appropriate measurements have been made.

\section{Acknowledoements}

In the course of this work we are profiting from the appreciated support of professionals from the interund from the Centre Suisse d'Electronique ot de Microand hniqu CSEM in Neuchatel, Switzarland Espocially, we would like to thank G. Declerck, I. Debusschere, R. Guye and 3. Jeannerat for many discussions.

The fruitful collaboration with the industrial partners Canberre Semiconductor N.V.. EnartecSchlumberger S.A. and Micron Ssmiconductor Ltd is acknowladged, and it la expocted that the conth
References

[1] 8.g. V.A.J. van Lint, G. Gigas and J. Barengoltz, Gilicon. IEEE Trans. Nucl. Sc. NS-22 (1975) 2663.

[2] J.Kemmer, P. Burger, R. Henck and E. Heijne, Performance and applications of passivated lon-implanted silicon detectors. FEEE Trens. Nucl. Sc. NS-29 (1982) 733

[3] The most comprehensive review of the oxide-silicon system can be found in: E.M. Nicollian and J.R. Brews. Technology" ${ }^{*}$. J. Willey \& Sons, Now Yark, 1982.

[4] J. Kemmer, Improvement of detector fabrication by the planar process, Nucl. Instr. Math. 226 (1982) 89.

[5] Z. Bian, J. Dobbins and N. Mistry. The uss of silicon photodiodes in a CoI(TI) calorimeter, Nucl. Instr. Math.

[6] Erik H.M. Heijne et al.o Development of hardware Nucl. Instr. Meth. 226 (1984) 63.

[7] Erik H.M. Heijne, Radiation damage: experience with sllicon detectors in high energy particle beams at CERN. procesdings Miniaturization of Detectors, Pisa Conference 1980. Ed. A. Steranini. Plenum Press, silicon dotectors in the CERN neutrino baems. CERN yellow raport 85-06, chapter 5 .

[8] B. Sigfridison and J.L. Linditröm, Electrical properties of electron-irradiated n-type sillicon, 3. Appl. Phys. 47 (1976) 4611 .

[9] H. Dietl, T. Gooch, R. Klenner. M. Pepé and $F$. Wickens, Radiation damage in silicon strip detectors, preprint, contribution to Euraphyse Conf. on High

[10] E.H.M. Heijne et al., A silicon surface barrier mlcrostrip detector dssigned for high enargy physics experiments, Nucl. Instr. Mieth. 178 (1980) 331. 


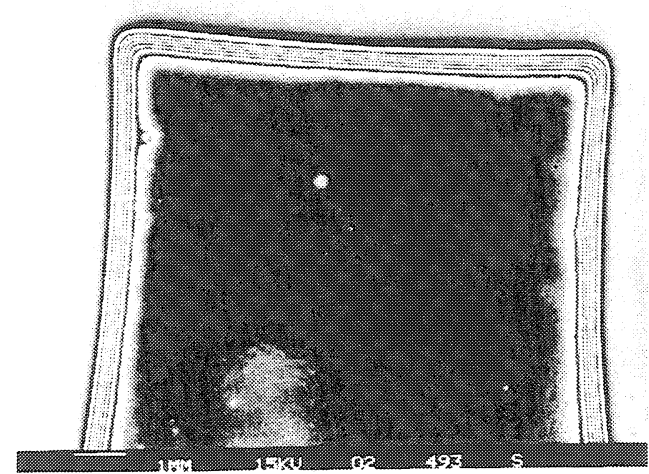

Fig. 17 EBIC picture of a large fraction of one segment on a 7-segment detector. The white spots are regions of bad collection of the EBIC signal, corresponding to regions of large carrierrecombination, and large leakage current.

Further study of these clearly visible defects might be possible, using elemental analysis with induced $X$-rays, ion-backscattering techniques, or other high resolution analysis methods.

From these first EBIC measurements it may already be concluded that large leakage currents are often caused by defects in the diode, rather than in the edges, although recombination in the edges and between segments is always higher than in the diode area. Both defects shown were probably existing already in the original wafer, although they may have been enlarged in processing.

\section{Conclusion}

Work has started in various directions to study problems which are encountered when planar detectors are used for large scale experiments. These problems generally do not preclude the use of these detectors for the aimed purpose, but longer lifetime, better performance and cost reduction could be achieved if the problems were solved.

Thanks to methods developed already in the field of microelectronics many of these problems may be understood, once appropriate measurements have been made.

\section{Acknowledgements}

In the course of this work we are profiting from the appreciated support of professionals from the Interuniversity MicroElectronics Center IMEC in Leuven. Belgium and from the Centre Suisse d'Electronique et de Microtechnique CSEM in Neuchatel. Switzerland. Especially, we would like to thank G. Declerck. I. Debusschere, R. Guye and J. Jeanneret for many discussions.

The fruitful collaboration with the industrial
Canberra Semiconductor N.V.s EnertecSchlumberger S.A. and Micron Semiconductor Ltd is acknowledged, and it is expected that the continuation of this work can help to establish excellent records for planar silicon detectors.

\section{References}

[1] e.g. V.A.J. van Lint, G. Gigas and J. Barengoltz, Correlation of displacement effects produced by electrons, protons and neutrons in silicon. IEEE Trans. Nucl. Sc. NS-22 (1975) 2663.

[2] J.Kemmer, P. Burger, R. Henck and E. Heijne, Performance and applications of passivated ion-implanted silicon detectors, IEEE Trans. Nucl. Sc. NS-29 (1982) 733.

[3] The most comprehensive review of the oxide-silicon system can be found in: E.H. Nicollian and J.R. Brews, "MOS (Metal Oxide Semiconductor) Physics and Technology". J. Wiley \& Sons, New York, 1982.

[4] J. Kemmer, Improvement of detector fabrication by the planar process, Nucl. Instr. Meth. 226 (1984) 89.

[5] Z. Bian, J. Dobbins and N. Mistry. The use of silicon photodiodes in a CsI(Tl) calorimeter, Nucl. Instr. Meth. A239(1985) 518.

[6] Erik H.M. Heijne et al., Development of hardware around a silicon microstrip detector with 512 elements, Nucl. Instr. Meth. 226 (1984) 63.

[7] Erik H.M. Heijne, Radiation damage: experience with silicon detectors in high energy particle beams at CERN, proceedings Miniaturization of Detectors, Pisa Conference 1980, Ed. A. Stefanini, Plenum Press, London 1983. Also in: Muon flux measurement with silicon detectors in the CERN neutrino beams, CERN yellow report $83-06$, chapter 5 .

[8] B. Sigfridsson and J.L. Lindström, Electrical properties of electron-irradiated n-type silicon, J. Appl. Phys. 47 (1976) 4611.

[9] H. Dietl, T. Gooch, R. Klanner, M. Pepé and F. Wickens, Radiation damage in silicon strip detectors preprint, contribution to Europhysics Conf. on High Energy Physics, Bari. 1985.

[10] E.H.M. Heijne et al., A silicon surface barrier microstrip detector designed for high energy physics experiments, Nucl. Instr. Meth. 178 (1980) 331. 